An analytical model of the vector digital magnetic recording process in isotropic thin media

This article has been downloaded from IOPscience. Please scroll down to see the full text article.

2001 J. Phys. D: Appl. Phys. 34879

(http://iopscience.iop.org/0022-3727/34/6/308)

View the table of contents for this issue, or go to the journal homepage for more

Download details:

IP Address: 144.173.204.17

The article was downloaded on 20/09/2012 at 13:16

Please note that terms and conditions apply. 


\title{
An analytical model of the vector digital magnetic recording process in isotropic thin media
}

\author{
B K Middleton, J J Miles and M M Aziz \\ Department of Computer Science, University of Manchester, Oxford Road, \\ Manchester M13 9PL, UK
}

Received 30 October 2000

\begin{abstract}
An analytical theory has been produced for describing the vector nature of the digital magnetic recording process in thin-film isotropic media. It is based upon assumptions on the way in which the magnetization follows the field during the recording process and shows, except for a scaling of their peak values, that the longitudinal and perpendicular components of magnetization behave, respectively, as if they were separately present in a longitudinal and perpendicular recording. The magnitudes of the longitudinal and perpendicular magnetization components are derived in particular cases.
\end{abstract}

\section{Introduction}

The digital magnetic recording process is complex. It involves a medium moving into the vicinity of a recording head and eventually away from the same. The field produced by the head varies in magnitude and direction as a function of position with respect to the middle of the head gap, and so the medium experiences a vector field history. The properties of the recording medium also vary with direction, thus making any analysis of the digital recording process difficult. To date, modelling of the 'vector' hysteresis process has only been accomplished to any satisfactory degree by employing numerical techniques with considerable complexity of programs and long computing times. A means of avoiding numerical complexity could be afforded by employing analytical modelling [1,2]. In both longitudinal and perpendicular recording simple models already exist and have enjoyed considerable success in explaining many observations $[1,2]$, but they effectively assume highly anisotropic media and ignore the components of magnetization which are not the primary concern. In longitudinal recording it is the shape anisotropy of the media that confines the magnetizations to the media planes, while in perpendicular recording it is the anisotropy within the substance of the media that produces the perpendicular orientations. In media where the anisotropy is not so strong and the magnetizations not so high, for example particulate media, both the longitudinal and perpendicular components of magnetization are known to be significant during recording. Here the simpler theories are least satisfactory and to be able to cover this type of situation vector modelling by analytical means remains in need of further development. Therefore, the purpose of this paper is to present a simple first step towards a more complete modelling by analytical means.

This paper considers media with hysteresis properties which are assumed to be the same in all directions and are termed isotropic. Poorly oriented particulate media approximate to this, and particulate media have also been purposely made with isotropic magnetic properties and high hysteresis loop squarenesses $[3,4]$; in these both the longitudinal and the perpendicular components of magnetization play a role during recording $[3,4]$. In addition, thin-film recording media of cobalt or cobalt alloys, used as disk recording media, often have grains exhibiting random distributions of easy axes. These media should be intrinsically isotropic when used for recording, but the shape anisotropy of the thin films discourages the perpendicular components of magnetization and the recording process is dominated by the longitudinal components. Nevertheless the perpendicular components are not entirely negligible. One of the purposes of this work is to investigate the contributions of both the longitudinal and the perpendicular components of magnetization to magnetic recording processes in intrinsically isotropic media and to explain these results analytically. The paper continues with a consideration of vector hysteresis and the recording process. 


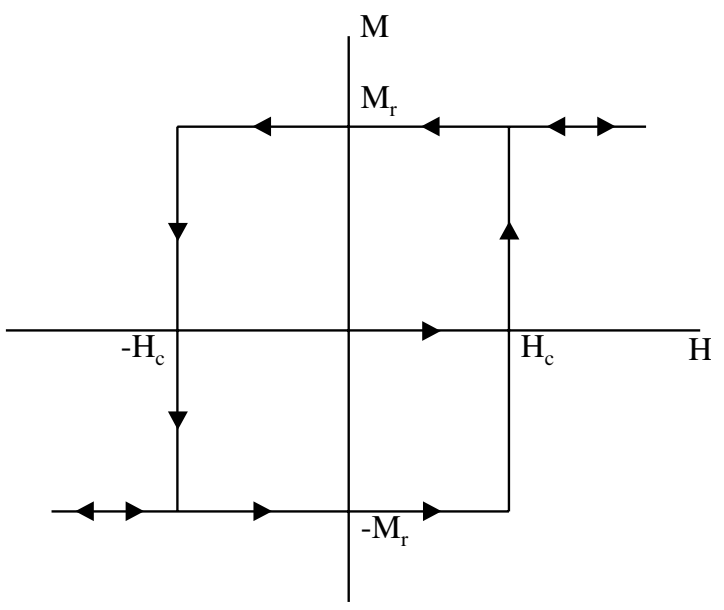

Figure 1. The assumed form of the hysteresis loop.

\section{Vector record theory}

\subsection{The vector hysteresis model: isotropic media}

To make progress in the modelling it is necessary to make some simplifying assumptions about the recording process. It is known that the application of a magnetic field serves to pull the magnetization towards alignment with the field. The underlying assumption in this work is that this tendency is complete and that changes of magnetization do create alignment of the magnetization and the field. In fact, detailed studies of recorded distributions reveal that the magnetization direction in the central region of a recorded transition does appear to follow closely the direction of the applied head field $[5,6]$. It is recognized that the above assumptions are an approximation to the reality where the alignment of the magnetization with the field is never perfect but the trend is clear. A recording model based upon this assumption is a valuable tool for investigating the vector nature of the recording processes.

This paper confines itself to a study of thin-film media of an isotropic nature with hysteresis loops, shown in figure 1, which are assumed assumed to be the same in all directions in the media. The general rules underlying the behaviour of the magnetization are as follows:

(a) for $-H_{c}<H<H_{c}$ the magnetization value or orientation is not altered by the applied fields;

(b) for $H= \pm H_{c}$ and $-M_{r}<M<M_{r}$ the magnetization can change and when it does it is to make $M$ parallel to $\boldsymbol{H}$;

(c) for $H>H_{c}$ then $M=M_{r}$ and for $H<-H_{c}$ then $M=-M_{r}$. This is saturation and $\boldsymbol{M}$ is parallel to $\boldsymbol{H}$.

As an example of the application of the above rules consider the following description of the magnetization processes in a previously demagnetized sample. Starting from the origin in figure 1 the application of an increasing field produces no effect, i.e. no magnetization, while $H<H_{c}$. When $H$ becomes equal to $H_{c}$, then $M$ takes on values from zero to $M_{r}$ with the magnetization pointing in the same direction as the applied field. When $H>H_{c}$ then $M=M_{r}$ and again the magnetization points in the same direction as the applied field. Any rotation of the applied field would cause an identical rotation of the direction of magnetization. On reducing the field from a large positive value the magnetization retains its value of $M_{r}$ and its parallelism with field as long as $H>H_{c}$. On reaching the value $H=H_{c}, M$ becomes frozen in direction and remains in that state until $H=-H_{c}$. M can then take values in the range $-M_{r}<M<M_{r}$, but aligns itself with the applied field. For $H<-H_{c} M$ takes the value $-M_{r}$ and is again parallel to the field. Increasing the field from the large negative value through to a large positive value then traces out the opposite side of the hysteresis loop.

\subsection{Head fields}

Typical contours of equal field produced by recording heads are shown in figure 2(a). It is through these that a recording medium moves to become magnetized. A medium moving along a surface at constant $y$ experiences a total field $H_{t}$, with components $H_{x}$ and $H_{y}$, which vary as shown in figure 3 . Reasonable approximations to the form of these fields for a narrow gap head are:

$$
\begin{gathered}
H_{t}=\frac{I}{\left(x^{2}+y^{2}\right)^{1 / 2}} \\
H_{x}=I \frac{y}{x^{2}+y^{2}} \\
H_{y}=-I \frac{x}{x^{2}+y^{2}}
\end{gathered}
$$

where

$$
H_{t}^{2}=H_{x}^{2}+H_{y}^{2} \text {. }
$$

Here $I=n i \eta ; n$ is the number of head turns, $\eta$ is the head efficiency, $i$ is the head current and $x$ and $y$ are defined in figure 2(a).

\subsection{Recording on demagnetized isotropic media}

2.3.1. Initial magnetization of the medium. Consider an initially demagnetized and thin medium moving from left to right under the recording head as shown in figure 2(a). If the peak of the total applied field $H_{t}$ is less than $H_{c}$ then the applied field is insufficient to magnetize the recording medium. The medium becomes magnetized when $H_{t}(0, y) \geqslant H_{c}$ and for values of $x>x_{-}$, where $x_{-}$is defined by

$$
H_{x}^{2}+H_{y}^{2}=H_{t}^{2}=H_{c}^{2} .
$$

Any demagnetizing fields have been neglected, since $M$ is small in this region. As an element of the medium moves past $x_{-}$it becomes magnetized and the magnetization grows and rotates to follow the field until, at $x=0$, where $H_{t}>H_{c}$, $M=M_{r}$. As the element moves to $x>0$ the magnetization vector rotates upwards to follow the direction of the field, but there comes a point $x_{o}$ where the total field has reduced to the coercivity and then the magnetization is fixed in magnitude and direction.

It is assumed, reasonably for this preliminary part of the magnetization process, that the recording medium is very thin and that planar components of demagnetizing field can be neglected. The $y$ component of demagnetizing field 


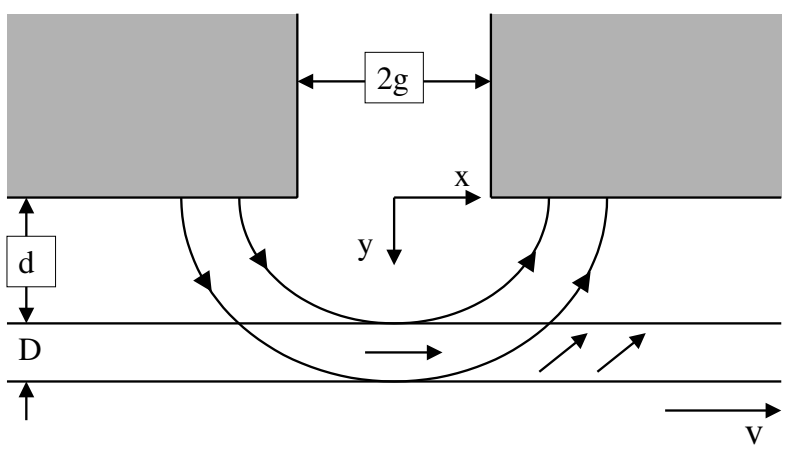

(a)

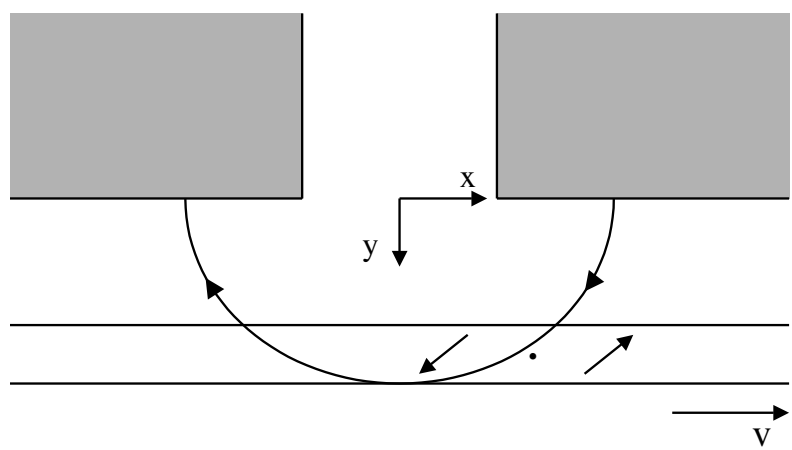

(b)

Figure 2. (a) Magnetization of a demagnetized medium prior to recording a transition. (b) The recording of a transition.

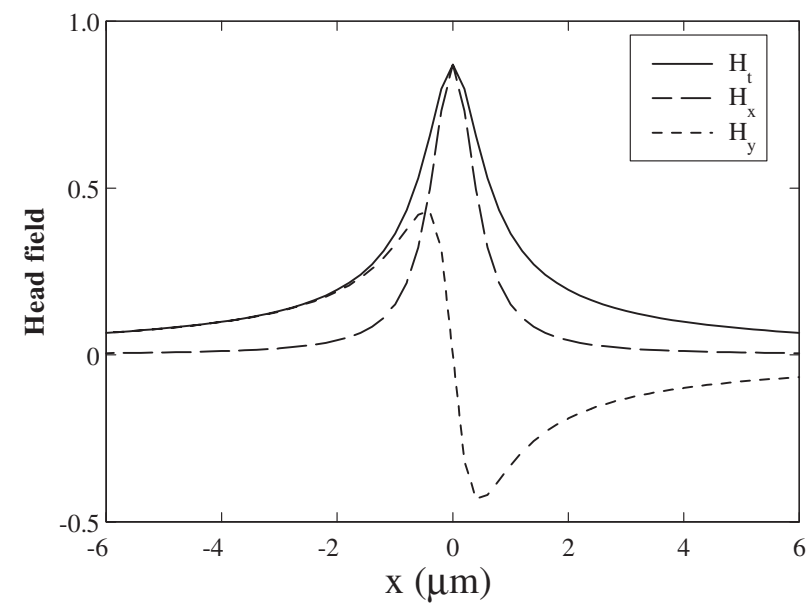

Figure 3. Total head field $H_{t}$ and its components $H_{x}$ and $H_{y}$ as functions of position below a recording head for $y=0.15 \mu \mathrm{m}$.

$H_{d y}\left(x_{o}\right)=-M_{y}\left(x_{o}\right)$ (i.e. the perpendicular demagnetizing factor is one), so that $x_{o}$ is defined by

$$
H_{x}^{2}\left(x_{o}\right)+\left(H_{y}\left(x_{o}\right)-M_{y}\left(x_{o}\right)\right)^{2}=H_{c}^{2} .
$$

At the same time the $x$ and $y$ components of magnetization, $M_{x o}$ and $M_{y o}$, are given by

$$
M_{x o}^{2}+M_{y o}^{2}=M_{r}^{2} .
$$

Noting that the field and magnetization are parallel, the manipulation of these two equations leads to the following, which defines $x_{0}$ :

$$
H_{x}^{2}\left(x_{o}\right)+H_{y}^{2}\left(x_{o}\right)\left(\frac{1}{1+M_{r} / H_{c}}\right)^{2}=H_{c}^{2}
$$

with expressions for $M_{x o}$ of

$$
M_{x o}=\frac{M_{r}}{H_{c}} H_{x}\left(x_{o}\right)
$$

and for $M_{y o}$ of

$$
M_{y o}=\frac{M_{r} / H_{c}}{1+M_{r} / H_{c}} H_{y}\left(x_{o}\right) .
$$

The values of $M_{x o}$ and $M_{y o}$ are the levels of magnetization created prior to switching of the head field and represent the levels of magnetization on either side of the transitions that are eventually to be written.

2.3.2. Recording of a transition. Figure 2(b) shows the situation when the head field is reversed and a transition is written. Inside the transition where $M$ changes, it must be for a rectangular hysteresis loop that

$$
\left(H_{x}+H_{d x}\right)^{2}+\left(H_{y}+H_{d y}\right)^{2}=H_{c}^{2} .
$$

Differentiating equation (8) gives

$\left(H_{x}+H_{d x}\right)\left(\frac{\mathrm{d} H_{x}}{\mathrm{~d} x}+\frac{\mathrm{d} H_{d x}}{\mathrm{~d} x}\right)+\left(H_{y}+H_{d y}\right)\left(\frac{\mathrm{d} H_{y}}{\mathrm{~d} x}+\frac{\mathrm{d} H_{d y}}{\mathrm{~d} x}\right)=0$.

In (8), the field $\left(H_{x}+H_{d x}\right)$ is negative and its gradient positive, while $\left(H_{y}+H_{d y}\right)$ is positive and its gradient is negative or zero. Equation (8) cannot be satisfied unless simultaneously

$$
\begin{aligned}
& \left(\frac{\mathrm{d} H_{x}}{\mathrm{~d} x}+\frac{\mathrm{d} H_{d x}}{\mathrm{~d} x}\right)=0 \\
& \left(\frac{\mathrm{d} H_{y}}{\mathrm{~d} x}+\frac{\mathrm{d} H_{d y}}{\mathrm{~d} x}\right)=0 .
\end{aligned}
$$

These are the defining equations for the magnetization distributions in the transitions and are those needing to be solved to learn about the details of transition. These equations have been obtained separately for longitudinal and perpendicular recording [1] and in combination they now bring together the contributions of the longitudinal and perpendicular components of magnetization to the recording process in isotropic media.

The exact solution of $(9 a)$ and $(9 b)$ is by numerical means, but the following section gives approximate solutions in terms of 'arctangent' theories.

2.3.3. An approximate solution. The recorded magnetization $M$ varies with position through the transition and at its centre $M=0$. Thus both the longitudinal and perpendicular components of magnetization go through zero at the same point. In recognition of this, and as in most simplified theories, it is assumed that the components of magnetization are given by

$$
M_{x}=\frac{2 M_{x o}}{\pi} \tan ^{-1}\left(\frac{x-x_{c}}{a_{x}}\right)
$$




$$
M_{y}=\frac{-2 M_{y o}}{\pi} \tan ^{-1}\left(\frac{x-x_{c}}{a_{y}}\right)
$$

where $x_{c}$ is the location of the centre of the transition. The demagnetizing fields at the centres of transitions with such symmetry are zero and the locations $x_{c}$ are then determined from

$$
H_{x}^{2}\left(x_{c}\right)+H_{y}^{2}\left(x_{c}\right)=H_{t}^{2}=H_{c}^{2}
$$

which, using $(1 a)$, leads to

$$
x_{c}=\left(\frac{I^{2}}{H_{c}^{2}}-y^{2}\right)^{1 / 2}
$$

where $y$ takes the value $(d+D / 2), d$ is the head to medium spacing and $D$ is the medium thickness, assumed to be small. To calculate $a_{x}$ and $a_{y}$ using the basic methodology of Williams and Comstock [7] it is necessary to solve $(9 a)$ and $(9 b)$ at the centre of the transition. Applying $(9 a)$ and $(9 b)$ in the usual way [1] in a thin medium leads to

$$
\begin{aligned}
& \left(\frac{\mathrm{d} H_{x}}{\mathrm{~d} x}\right)_{x_{c}}-\frac{1}{2 \pi} \frac{M_{x o} D}{a_{x}^{2}}=0 \\
& \left(\frac{\mathrm{d} H_{y}}{\mathrm{~d} x}\right)_{x_{c}}-\frac{2}{\pi} \frac{M_{y o}}{a_{y}+D / 2}=0
\end{aligned}
$$

and then the transition width parameters are

$$
\begin{aligned}
& a_{x}=\left(\frac{M_{x o} D}{2 \pi\left(\mathrm{d} H_{x} / \mathrm{d} x\right)_{x_{c}}}\right)^{1 / 2} \\
& a_{y}=\frac{2 M_{y o}}{\pi\left(\mathrm{d} H_{y} / \mathrm{d} x\right)_{x_{c}}}-\frac{D}{2} .
\end{aligned}
$$

The head field gradients used above are obtained from the gradients of $(1 a)$ and $(1 b)$ at $x=x_{c}$ for the appropriate value of the recording current. Equations (15) and (16) are the usual expressions for the transition width parameters, except that the field gradients are slightly modified and the values of $M_{x o}$ and $M_{y o}$ are both reduced below $M_{r}$ in accordance with ( $6 a$ ) and $(6 b)$. These results indicate that the behaviour of isotropic media can be closely approximated using the independently produced recording theories for longitudinal and perpendicular recording but subject to the scaling of the magnitude of the magnetization components.

\section{Results and discussion}

Figure 4 shows the reduced values of the longitudinal and perpendicular magnetization components from equation (5) as functions of $M_{r} / H_{c}$ for values of the recording current of $\sqrt{2} H_{c} y$ and $2 H_{c} y$. They clearly indicate that perpendicular components are large only at small values of $M_{r} / H_{c}$ and in many cases will contribute little to the net output. For $M_{r} / H_{c}=2.09$, which corresponds to a thin-film disk recording, and a peak record head field $H_{t}=2 H_{c}$, $M_{x o}=0.987 M_{r}$ and $M_{y o}=0.158 M_{r}$. These contribute to the total output pulse as shown in figure 5 and this is very much in line with the general experience on thin-film disk media where there is only a small contribution from the perpendicular components of magnetization [1,2]. Elsewhere it has also been confirmed that the location of the recorded transition is

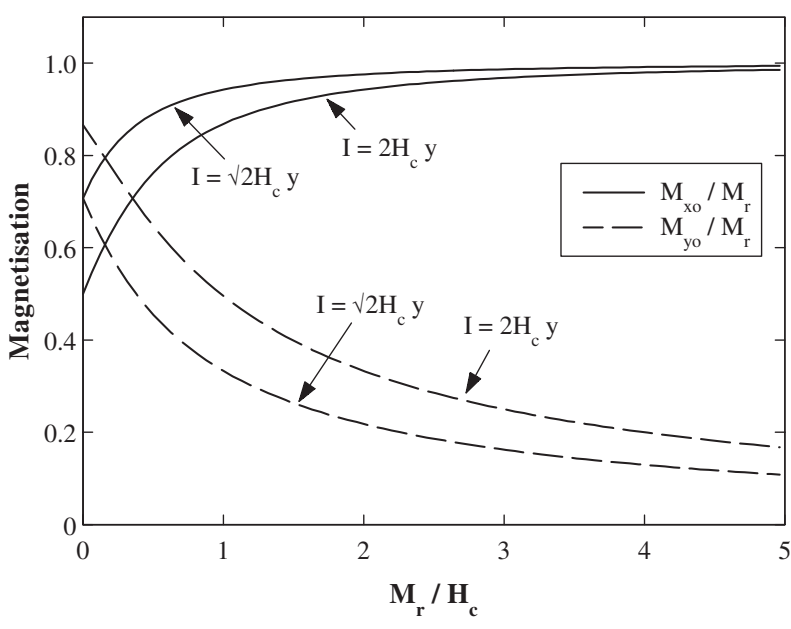

Figure 4. Reduced values of the longitudinal and perpendicular components of magnetization as functions of $M_{r} / H_{c}$ for different recording currents. The film coercivity is $159.2 \mathrm{kA} \mathrm{m}^{-1}$ and $d=0.15 \mu \mathrm{m}$.

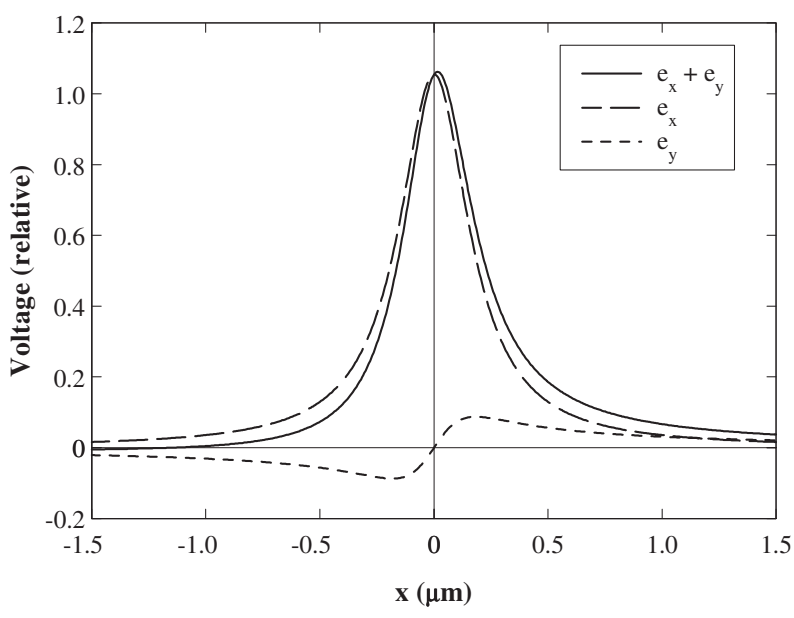

Figure 5. The output pulse for $I=2 H_{c} d$ and contributions to it from the longitudinal and perpendicular components of magnetization for a thin-film recording medium with $M_{r}=333 \mathrm{kA} \mathrm{m}^{-1}, H_{c}=159 \mathrm{kA} \mathrm{m}^{-1}, D=30 \mathrm{~nm}$ and $d=0.15 \mu \mathrm{m} . a_{x}$ and $a_{y}$ were calculated to be $0.07 \mu \mathrm{m}$ and $0.08 \mu \mathrm{m}$ respectively.

determined by the total head field (5), as shown here, rather than just the longitudinal component [8].

Experimental studies have been carried out on thick particulate media [3] and while the current theory, involving thin films, has limited applicability here there are a number of general points which can be made. First, it is clear from the work of Lemke [3], on isotropic particulate media of low magnetization, that $\boldsymbol{M}$ closely follows $\boldsymbol{H}$ as its direction is varied, in line with the basic assumption of this paper. Second, it was shown that the ratio $M_{x o} / M_{y o}$ decreased on increasing the recording current [3] as is implied in figure 6, which shows separately the variations of $M_{x o}$ and $M_{y o}$ as functions of recording current. This result has also been implied elsewhere [9].

Figure 6 also shows the effect of the head-to-medium spacing on the longitudinal and perpendicular components of magnetization for different values of $M_{r} / H_{c}$. Increasing the spacing increases $M_{x o}$ and reduces $M_{y o}$, and vice versa. 


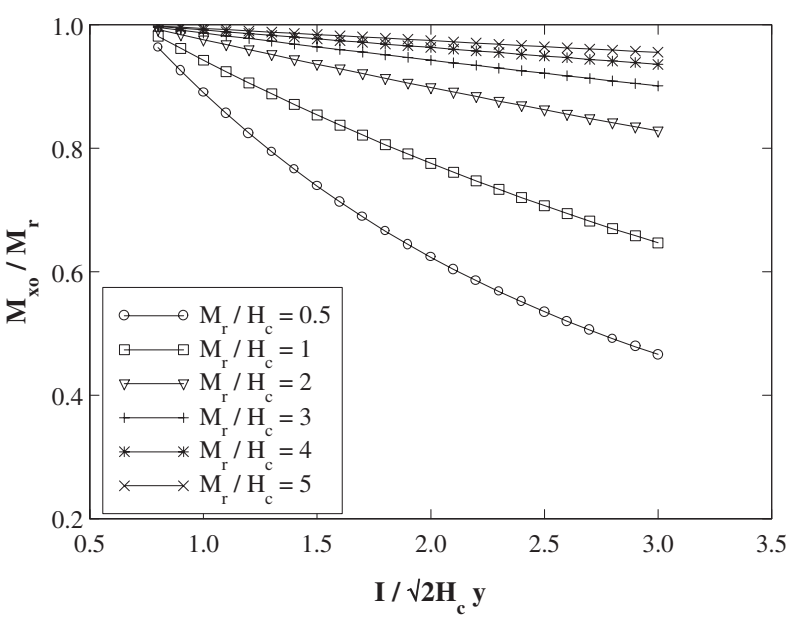

(a)

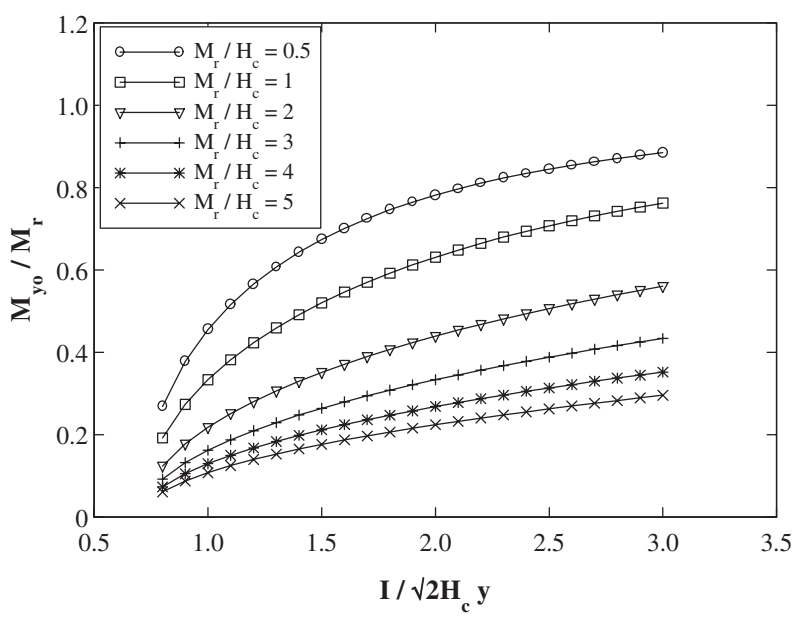

(b)

Figure 6. Reduced values of magnetization as functions of the recording current for different values of $M_{r} / H_{c}$, again for $d=0.15 \mu \mathrm{m}$ and $H_{c}=159.2 \mathrm{kA} \mathrm{m}^{-1}$.

This is in line with the observations of Lemke [3,4] who found high values of $M_{y o}$ at low head-to-medium spacings. In the particular case when $M_{r} / H_{c}=0$, recording is demagnetization free and there are no interactions through the depths of the media. Then (5), (6a) and ( $6 b)$ can easily be used to show that the variation of magnetization with depth $y$ into a thick medium is given by

$$
\frac{M_{x o}}{M_{r}}=\frac{y}{N d}
$$

and

$$
\frac{M_{y o}}{M_{r}}=\left(1-\frac{y^{2}}{N^{2} d^{2}}\right)^{1 / 2}
$$

where $N=I / H_{c} d$. This shows the same trends as the computations of Bertram [5] and Otter [10]. The low values of $M_{x o}$ for small $y$ show up as low surface values of magnetization in simulations of the digital recording process in thick particulate media [5] where interactions are low.

\section{Conclusions}

A preliminary theory of digital recording in isotropic media has been produced which shows a general affinity with experimental observation and with computations cited in the literature. Furthermore, it has been shown that recorded transitions can reasonably be represented by the sum of the longitudinal and perpendicular magnetizations calculated separately using longitudinal and perpendicular recording theories but modified by a scaling of the magnetizations. This is in general sympathy with the approach used successfully in the numerical computations of Ortenburger et al [11].

The current theory is applicable to isotropic media with rectangular hysteresis loops but could be adapted to nonrectangular loops whose shapes vary with the direction of the applied field. Such a development involves no change of principle, but would be at the expense of algebraic simplicity. The shortcoming of the theory is that while it takes into account the odd Fourier components of the perpendicular components of magnetization, it does not cater for the even components, which are well known in the transitions recorded in particulate media [10].

\section{Acknowledgment}

Thanks are due to EPSRC for studentship (M Aziz) and grant support.

\section{References}

[1] Middleton B K 1996 Recording and reproducing processes Magnetic Recording Technology ed C D Mee and E D Daniel (New York: McGraw-Hill) ch 2

[2] Middleton B K 1991 Models of the longitudinal digital magnetic recording processes in thin films IEEE Trans. Magn. 27 3563-9

[3] Lemke J U 1982 An isotropic particulate medium with additive Hilbert and Fourier field components J. Appl. Phys. $532561-6$

[4] Lemke J U 1979 Ultra high density recording with new heads and tapes IEEE Trans. Magn. 15 1561-3

[5] Bertram H N 1984 Geometric effects in the magnetic recording process IEEE Trans. Magn. 20 468-78

[6] Fayling R E 1980 Edge profile studies of recorded flux transitions IEEE Trans. Magn. 16 1249-54

[7] Williams M L and Comstock R L 1971 An analytical model of the write process in digital magnetic recording Proc. 17th Ann., AIP Conf. vol 5, pp 738-42

[8] Lee J E and Truman N N 1968 The effect of vertical head field component on the switching of thin cobalt films IEEE Trans. Magn. 4 277-80

[9] Li Hui Hong J K, Middleton B K and Miles J J 1993 Modelling recording system performance and its relationship to recording media properties J. Magn. Magn. Mater. 120 206-9

[10] Otter E L 1988 Write saturation current as a function of tape magnetisation IEEE Trans. Magn. 24 2485-7

[11] Ortenburger I B, Cole R W and Potter R I 1977 Improvements to a self-consistent model for the magnetic recording properties of non-particulate media IEEE Trans. Magn. 13 $1278-83$ 